for pediatricians. Pediatric Pulmonol. 2020;55:E1-E4.

3. Sun D, Li H, Lu X, Xiao H, Ren J, Zhang FR, et al. Clinical features of severe pediatric patients with coronavirus disease 2019 in Wuhan: A single center's observational study. World J Pediatr. 2020 Mar 19: 1-9. Available from: https://www.ncbi.nlm.nih.gov/pmc/articles/PMC7091225. Accessed April 26, 2020.

4. Colson P, Rolain JM, Raoult D. Chloroquine for the 2019 novel coronavirus SARS-CoV-2. Int J Antimicrob Agents. 2020;55:105923.

5. Ponticelli C, Moroni G. Hydroxychloroquine in systemic lupus erythematosus (SLE). Expert Opin Drug Saf. 2017;16:411-9.

6. Bai Y, Yao L, Wei T, Tian F, Jin DY, Chen L, et al. Presumed asymptomatic carrier transmission of COVID-19. JAMA. 2020;323:1406-7.

\section{Demystifying BCG Vaccine and COVID-19 Relationship}

Efforts for developing vaccines for novel coronavirus disease (COVID-19) are ongoing, but it is unlikely to be available in the immediate future [1]. In the absence of specific therapy, the researchers are exploring other potential preventive and therapeutic options. Recently, there has been a buzz about the protective effect of Bacille Calmette-Guérin (BCG) vaccine in COVID-19. Based on epidemiological correlations, many unpublished preprints hypothesized that the BCG vaccine may offer protection against COVID-19. It gained so much popularity that within 20 days three randomized controlled trials (RCTs) got registered, and many more are in the pipeline [2]. To make an informed decision, we must understand the mechanism of action of BCG, and appraise the robustness of the evidence.

The basis of the possible use of the BCG vaccine against COVID-19 lies in its non-specific effects (NSEs) over the immune system [3]. The NSEs of BCG are mainly mediated by potentiating innate immune response through epigenetic mechanisms. These epigenetic changes within the innate cells act as de novo enhancers to boost the immune response against a secondary challenge [3-5]. This enhancing response is popularly known as 'trained immunity' and is very characteristic of BCG. This trained immunity also offers protection against a variety of pathogens (Salmonella, Shigella, malaria, respiratory viruses, etc.) other than Mycobacterium tuberculosis, and forms the basis of its use in bladder cancer, melanoma etc. However, this non-specific effect is mostly short-lived and wanes soon after the primary BCG stimulus is cleared from the body. By virtue of the NSEs, BCG vaccine has shown to decrease all-cause mortality in children. Though a few observational studies suggest that the NSEs may last till adulthood, but the overall evidence is still inadequate and is of low quality $[3,6,7]$.
On critical appraisal of the non-peer reviewed preprint evidence, at the relationship between BCG and COVID-19 is being proven by looking at correlation/ association among two data set (BCG vaccine coverage and COVID-19), without acknowledging the confounders. The variables like the difference in testing strategies, reporting bias, demographics, nation's ability to respond to the pandemic, prevalence of co-morbidities, and different stages of the pandemic across various countries might have a significant impact on these associations/correlations and must be interpreted carefully. Therefore, at this stage, this association should be considered as a hypothesis only and should be tested through appropriately designed studies.

Though the epidemiological association between BCG and COVID-19 is striking, it does not prove causal relationship unless tested in well-designed clinical trials. Also, we should not forget that the NSEs of the BCG vaccine has not been well-studied in human beings and their clinical relevance is unknown [2,3]. Therefore, in the absence of evidence, the BCG vaccination for the prevention of COVID-19 cannot be recommended. The results of the ongoing RCTs shall guide us further.

Funding: Alone, Competing interest: None Stated. Published online: April 30, 2020; PII: S097475591600168

$$
\begin{array}{r}
\text { JOGENDER KUMAR }{ }^{1 *} \text { AND JITENDRA MEENA }{ }^{2} \\
\text { Departments of Pediatrics, } \\
{ }^{1} \text { Post Graduate Institute of Medical Education and Research, } \\
\text { Chandigarh; and }{ }^{2} \text { All India Institute of Medical Sciences, } \\
\text { New Delhi; India. } \\
{ }^{* J o g e n d r a y a d v @ g m a i l . c o m}
\end{array}
$$

\section{REFERENCES}

1. Ella KM, Mohan KV. Coronavirus vaccines: Light at the end of the tunnel. Indian Pediatr. 2020 Apr 15 [Epub] Available from: https://www.indianpediatrics.net/COVID29.03.2020/ PERS-00163.pdf. Accessed April 26, 2020.

2. World Health Organization. Bacille Calmette-Guérin (BCG) vaccination and COVID-19. Available from: $h t t p s: /$ /www.who.int/news-room/commentaries/detail/ bacillecalmette-guérin-(bcg)-vaccination-and-covid-19. 
3. SAGE Working Group on BCG Vaccines and WHO Secretariat. Report on BCG vaccine use for protection against mycobacterial infections including tuberculosis, leprosy, and other nontuberculous mycobacteria (NTM) infections. World Health Organization; 2017. Available from: https://www.who.int/immunization/sage/ meetings/ 2017/october/1_BCG_report_revised_version_online.pdf. Accessed April 14, 2020.

4. Moorlag SJCFM, Arts RJW, van Crevel R, Netea MG. Non-specific effects of BCG vaccine on viral infections. Clin Microbiol Infect. 2019;25:1473-8.

5. Arts RJW, Moorlag SJCFM, Novakovic B, Li Y, Wang SY, Oosting $\mathrm{M}$, et al. BCG vaccination protects against experimental viral infection in humans through the induction of cytokines associated with trained immunity. Cell Host Microbe. 2018;23:89-100.e5.

6. Usher NT, Chang S, Howard RS, Martinez A, Harrison LH, Santosham M, et al. Association of BCG vaccination in childhood with subsequent cancer diagnoses: A 60-Year Follow-up of a clinical trial. JAMA Network Open. 2019;2:e1912014.

7. Abbott S, Christensen H, Lalor MK, Zenner D, Campbell $\mathrm{C}$, Ramsay ME, et al. Exploring the effects of BCG vaccination in patients diagnosed with tuberculosis: Observational study using the Enhanced Tuberculosis Surveillance system. Vaccine. 2019;37:5067-72.

\section{COVID -19 Pandemic: The Challenges for Pediatric Oncology}

The global pandemic of the novel coronavirus disease (COVID-19) has had a significant impact on adult and pediatric patients with many acute and chronic diseases including cancer. The purpose of this correspondence is to project the challenges faced by both, the children undergoing treatment and the treating pediatric oncologist [1].

Providing medical care to children with cancer during this pandemic is challenging given the risks of death from cancer versus death or serious complications in the immunocompromised hosts [2,3]. Hospitals are delaying chemotherapy, radiotherapy and surgery after being overwhelmed by COVID-19 infection. Patients with cancer also fear coming to hospital fearing the risk of infection. There are also limited supplies of personal protective equipment (PPE) for doctors and other personnel, limited beds and ICU facility, limited blood bank facilities and also strained diagnostic facilities. Limited data, though from adults, also suggests that cancer patients with COVID will fare worse [4]. In a study from China, there was a higher risk of severe events in COVID-19 patients with cancer compared with those without cancer. Theoretically, immune therapy can result in cytokine release and worsen the viral injury, which is also believed to be due to a cytokine storm, but this is a theoretical consideration and not proven due to very limited data.

Some general guidelines have been provided for children with cancer [5], which include, in addition to social distancing, mask usage outside home and hygiene, viz. clinic visits that can be postponed without risk to the patient should be postponed and telemedicine to be used to screen and evaluate patients. Certain other issues are briefly elucidated herein.

Difficulty in hospital consultation: Many hospitals have been designated as centers for management of COVID-19, leading to temporary stoppage of outpatient services. Children with malignancies are unable to come for outpatient consultation for clinical follow-up and chemotherapy planning. In addition, it is now difficult for outstation patients to visit referral centers, despite prior appointments.

New cases needing evaluation are also not able to reach some of the major hospitals, which have temporarily closed OPD registrations to focus on COVID patients. There is also risk of new patients being asymptomatic carriers, but the current guidelines do not allow COVID testing for all new hospital admissions even if they are immune-compromised cancer patients.

Increased risk of coronavirus infection: The pandemic poses a risk of coronavirus infection for all individuals including children. Children with cancer are assumed to be more susceptible to the coronavirus due to inherent suppressed immune function associated with cancer treatment and repeated attendance in health care facilities [2].

Treatment delay: Children with cancer, already on treatment, were advised to stay indoors and practice social distancing resulting in delay in their treatment. The nationwide lockdown further delayed the treatment due to restricted mobility even within the city or travel from outstation. The bed strength available for in-house admission has also reduced due to diversion to COVID-19 wards, leading to delayed/deferred intensive chemo- 